\title{
DEVELOPMENT OF A PORTABLE PAPER-BASED MICROFLUIDIC DEVICE FOR THE DETECTION OF ALCOHOL IN BIOLOGICAL FLUIDS
}

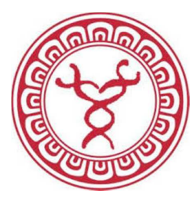

Gunawardhana D.Y.R. and Kaumal M. N.*

Department of Chemistry, University of Colombo, Colombo 03, Sri Lanka.

\section{Abstract}

A paper-based microfluidic device with Agaricus bisporus homogenate as the working electrode modifier was developed based on cyclic voltammetry for quantitative detection of ethanol in biological samples. Mushroom homogenate was mixed with gelatin and combined with carbon paste to prepare a slurry to develop the working electrode on paper. The homogenate contained alcohol oxidase enzyme that catalyzed the degradation of ethanol to acetaldehyde and hydrogen peroxide in the presence of oxygen. A graphite crystalline pencil (5HB) was used to draw the counter electrode and a silver conductive ink pen was used to draw the reference electrode on the paper. Assay is based on the detection of reduction peaks of remaining oxygen at the working electrode using cyclic voltammetry at potentials between +0.4 and $+0.8 \mathrm{~V}$. The expected electrode response showed a linier relationship for ethanol concentrations ranging from 4.0 to $14.0 \mathrm{mM}$. Phosphate buffer $(\mathrm{pH}=7)$ was used as the supporting electrolyte and it also provided the optimal condition for the activity of alcohol oxidase enzyme extracted from the mushroom tissue. The proposed paper-based device was validated using laboratory prepared samples.

Key words: alcohol, cyclic voltammetry, disposable, paper-based microfluidic, portable 


\section{Introduction}

Levels of ethanol in body fluids are required to be tested mainly due to the toxicity of alcohol and for legal purposes. For medical proposes it is essential to determine the level of ethanol in blood in order to effectively treat the people intoxicated with ethanol. Even though there are many methods, breathalyzer is the most common method used for the determination of ethanol level in blood indirectly. Although breathalyzer is a quick and economical method, its accuracy can vary based on environmental factors such as humidity and temperature (Simpson, 1987).

Paper-based microfluidic devices ( $\mu$ PADs) are getting popular among scientists as an alternative platform to develop many economical one-time-use biosensors (Cate et al., 2015) because they provide power-free fluid transport via capillary action, have a higher surface area to volume ratio, and can store reagents in active form within the fiber network (Cate et al., 2015). Further, they are less expensive, portable, light weight, easily available, compatible with biological samples, and can easily be disposed of after single use (Cate et al., 2015).

The $\mu P A D$ s are developed using commonly available filter paper. The three electrode system is fabricated using commonly available materials. The spherical shape electrochemical reaction region is constructed using commercially available varnish paint. This paper-based system has never been used for the detection of alcohol. The working electrode is modified with alcohol oxidase (AOX) enzyme originated from Agaricus bisporus mushroom. Use of Agaricus bisporus mushroom homogenate for the extraction of AOX is previously reported for the alcohol determination (Akyilmaz et al., 2005). The developed electro chemical device is shown in Figure 1. AOX can oxidize ethanol in the presence of oxygen, and this reduces the amount of oxygen in the sample. The reaction between alcohol and oxygen as follows.

Alcohol + Oxygen $\underset{\text { AOX }}{\longrightarrow}$ Aldehyde $+\mathrm{H}_{2} \mathrm{O}_{2}$

The amount of oxygen in the sample depends on the level of alcohol in the sample due to the above reaction between alcohol and oxygen in the presence of AOX. The amount of alcohol can be estimated based on the oxygen concentration in the sample in the presence of AOX.

Cyclic voltammetry is used to determine the remaining oxygen level in the sample. Cyclic voltammetry produces quick and accurate results compared to other analytical methods. Biosensor developed based on $\mu P A D$ s is suitable to work with various body fluids since this sensor required small sample volumes to produce a satisfactory measurement (Cate et al., 2015).

\section{Material and Methods}

\subsection{Reagents and solutions}

Agaricus bisporus, varnish and gelatin were purchased commercially and mushroom was stored at $4{ }^{\circ} \mathrm{C}$ until use. Absolute ethanol (Hayman Ltd UK), $\mathrm{NaCl}$ (Fischer chemicals ltd), $\mathrm{KCl}$ (Fischer chemicals ltd), $\mathrm{Na}_{2} \mathrm{HPO}_{4}$ (Merck chemicals ltd), $\mathrm{KH}_{2} \mathrm{PO}_{4}$ (BDH chemicals ltd), $\mathrm{HCl}$ (Fluka) $\mathrm{K}_{2} \mathrm{Fe}(\mathrm{CN})_{6} \cdot 3 \mathrm{H}_{2} \mathrm{O}$ (BDH chemicals

*Corresponding author: Tel: 0773311010; E-mail: mnkaumal@sci.cmb.ac.lk 
ltd) and $\mathrm{KCl}$ (Fluka) were used as they were received. Binder was prepared using phenol (BDH chemicals ltd), formaldehyde (Fluka Chemicals) and oxalic acid (BDH chemicals ltd). Graphite powder, <20 $\mu \mathrm{m}$, synthetic (Sigma chemicals) was used to fabricate the working electrode.

\subsection{Instruments}

Pine WaveDriver 10 potentiostat/ galvanostat along with computer based AfterMath software was used to perform all cyclic voltammetric experiments.

\subsection{Fabrication of $\mu P A D s$}

Whatman No.01, varnish, silver conductive pen, 5B finest crystalline graphite pencil were used for the fabrication of $\mu P A D$ s.

\subsection{Preparation of working electrode}

Mushroom (A. bisporus) tissue (200 g) was homogenized in phosphate buffer (200 mL, pH = 7) using a motar and a pestle. Filtered homogenate was then mixed with gelatin ( $5 \mathrm{~g}$ ) and incubated at $38{ }^{\circ} \mathrm{C}$ for $5 \mathrm{~min}$ to dissolve gelatin. This preparation was stored at $4{ }^{\circ} \mathrm{C}$ until use. A $50 \mu \mathrm{l}$ portion of this mixture was combined with $5 \mathrm{~g}$ of graphite powder and phenol formaldehyde resin to provide a slurry to draw the electrode on the paper-based device.

\subsection{Fabrication of $\mu P A D s$}

Varnish was used to draw the spherical electrochemical reaction region on the Whatman 01 paper as shown in the Figure 1. A silver conductive pen and a $5 \mathrm{HB}$ pencil were used to fabricate the pseudo-reference and auxiliary electrodes, respectively.

\subsection{Methodology}

Each cyclic voltammetric run was performed after introducing $50 \mu \mathrm{L}$ on to the $\mu P A D$. Cyclic voltammetric analysis $(+1$ to $-1 \mathrm{~V}$, with the scan rate of $5,25,50$, $100,150 \mathrm{mV} / \mathrm{s}$ ) was carried out for standard ethanol solutions $(2,4,6,8,10$, $12,14,16,18$, and $20 \mathrm{mM}$ ) in $\mathrm{pH} 7$ phosphate buffered saline solutions. Same procedure was repeated with the laboratory prepared alcohol samples in saline buffered at $\mathrm{pH}=7$.

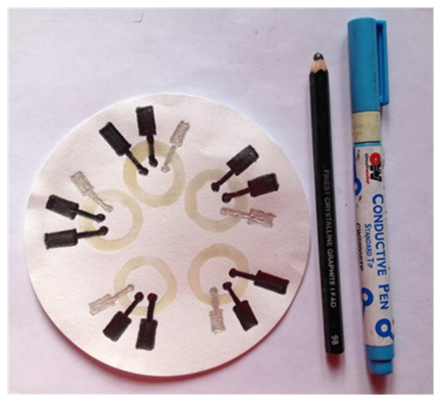

Figure 1. Five electrochemical paper-based devices developed on a filter paper. The silver conductive ink pen and 5HB pencil were used for the development of auxiliary and reference electrodes respectively. The working electrode is modified with alcohol oxidase enzyme. 


\section{Results}

Response current variation with the scan rates $(5,25,50,100$, and $150 \mathrm{mV} / \mathrm{s})$ is shown in Figure 2 for saline solution buffered at $\mathrm{pH}=7$. Voltammograms collected for different standard ethanol solutions are shown in the Figure 3.

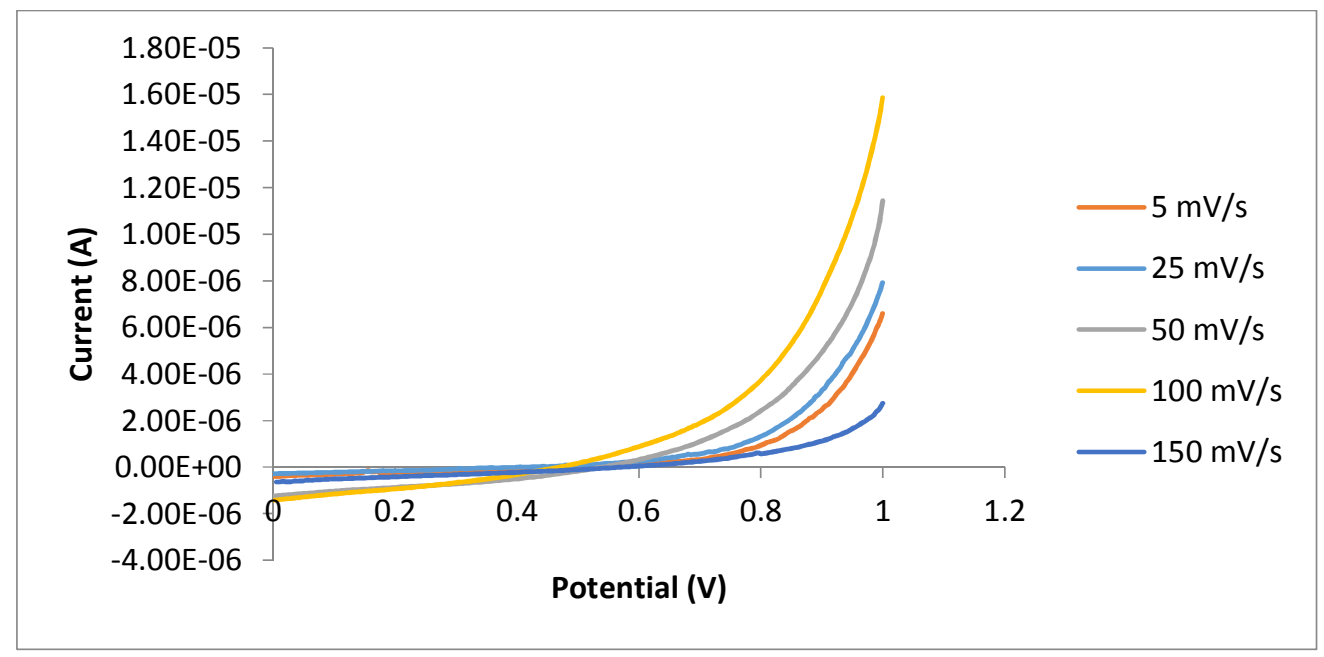

Figure 2. Response current at the scan rates of $5,25,50,100$, and $150 \mathrm{mV} / \mathrm{s}$ for saline solution buffered at $\mathrm{pH}=7$.

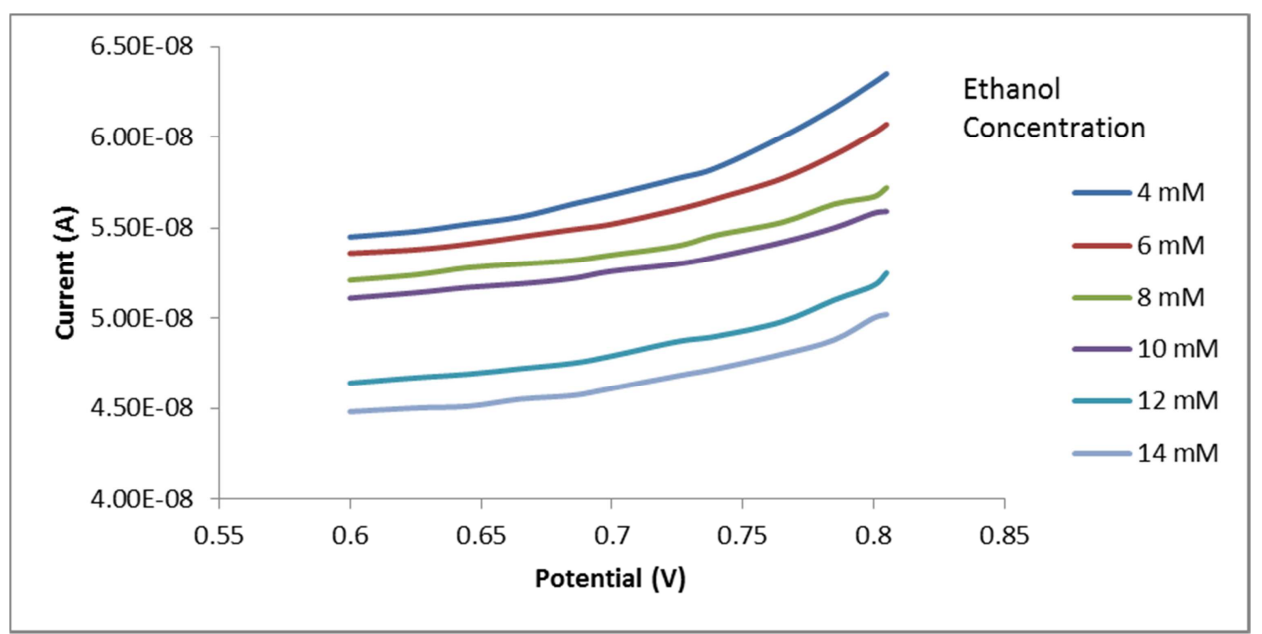

Figure 3. Response current for ethanol standards $(4,6,8,10,12$ and $14 \mathrm{mM})$ in saline buffered at $\mathrm{pH}=7$ collected at $25 \mathrm{mV} / \mathrm{s}$ scan rate. 


\section{Discussion}

As shown in Figure 2, at higher scan rates, the response start to decrease, so 25 $\mathrm{mV} / \mathrm{s}$ was selected as the optimum scan rate. With the increase of ethanol concentration, the amount of oxygen concentration in the sample solution was reduced due to oxidation reaction between oxygen and ethanol in the presence of AOX.

Currents at $0.6-0.8 \mathrm{~V}$ for the ethanol concentrations ranging from $4-14 \mathrm{mM}$ are shown in the Figure 3. The lowest current at a given potential is observed for the highest alcohol concentration, 14 $\mathrm{mM}$. The current response at various potentials from +0.6 to $+0.8 \mathrm{~V}$ were plotted and the best correlation was observed at +0.72 V. Alcohol concentration and the current response (calibration graph) at $+0.72 \mathrm{~V}$ is shown in the Figure 4. This graph shows a significant linier relationship $\left(\mathrm{R}^{2}=0.96\right)$ between the response current and alcohol concentration in the range 2 to $12 \mathrm{mM}$. The proposed device produced optimum results under these condition and the device is shown in Figure 5.

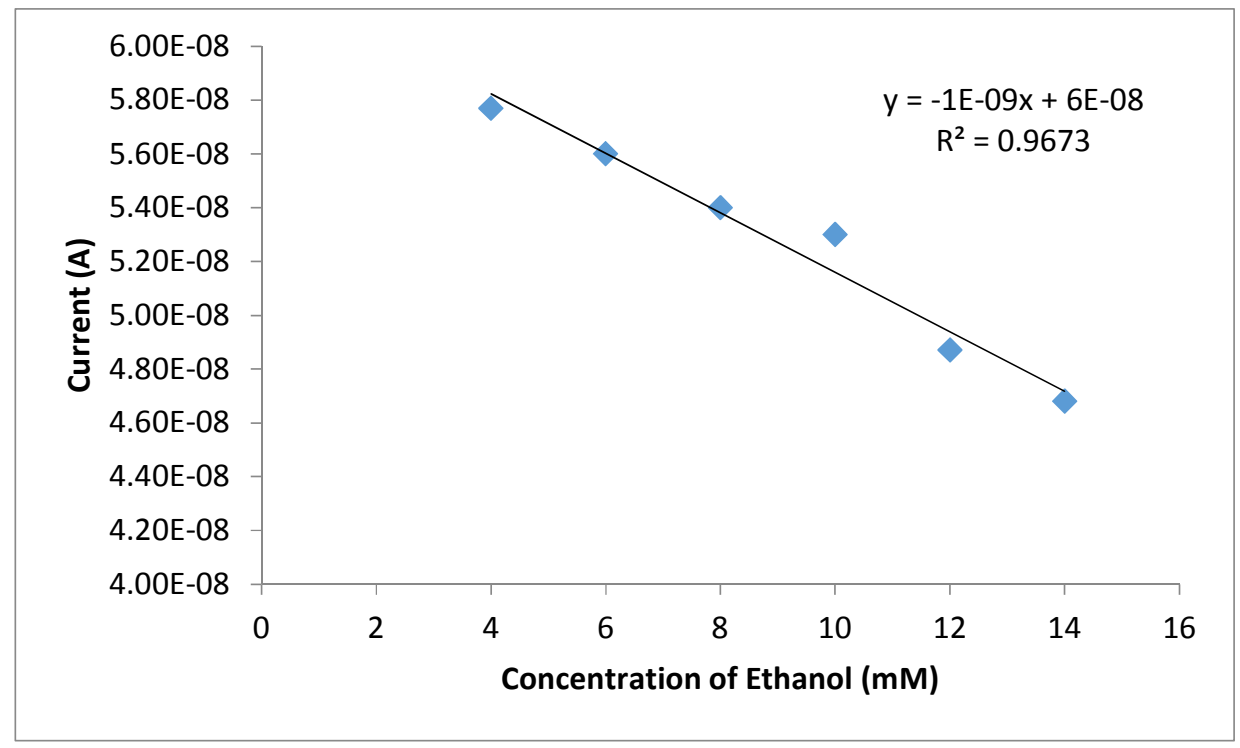

Figure 4. Response current collected at $0.72 \mathrm{~V}$ with the scan rate of $25 \mathrm{~V} / \mathrm{s}$ for ethanol standards $(4,6,8,10,12$ and $14 \mathrm{mM})$ in saline buffered at $\mathrm{pH}=7$.

The accuracy of the method was tested using laboratory prepared samples. Five laboratory prepared samples were tested with the developed biosensor and the experimental alcohol concentration was statistically compared with the actual concentration. There is no statistically significant difference between experimental results and the actual concentrations.

The lowest detection limit (LOD) was calculated using the calibration plot shown in Figure 4. Using the calibration plot, standard deviation of the slope $\left(S_{y}\right)$ and the slope (S) were calculated. The LOD, which was calculated using the relationship, $\mathrm{LOD}=3.3\left(\mathrm{~S}_{\mathrm{y}} / \mathrm{S}\right),(\mathrm{Wu}$ et al.,

*Corresponding author: Tel: 0773311010; E-mail: mnkaumal@sci.cmb.ac.lk 


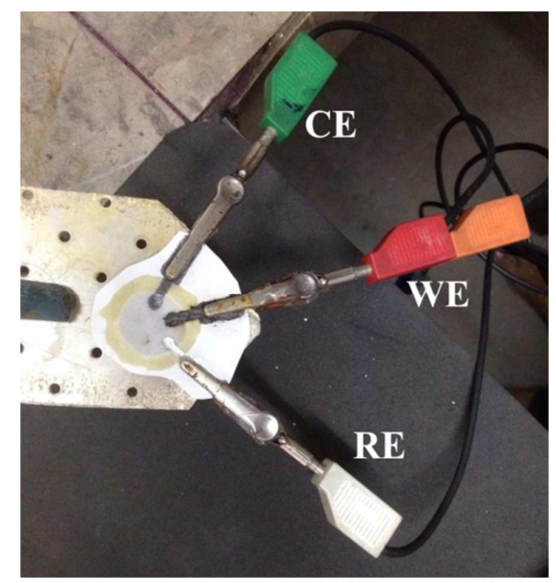

Figure 5. The proposed $\mu P A D$ device connected to the CE (counter electrode), WE (working electrode) and RE (reference electrode).

\section{Conclusion}

The proposed $\mu P A D$ can detect the level of alcohol in saline matrix with $95 \%$ confident and the lowest ethanol concentration that can be detected using the device is $2.8 \mathrm{mM}$. This novel, one time use paper-based devise is a potential tool that can be used successfully to detect alcohol in saliva. Connected to a portable potentiostat, this $\mu P A D$ can be used as a portable alcohol biosensor. This one time use economical device eliminate the cross contamination and assure the safety of the consumer.

\section{References}

Akyilmaz, E., Imer, O. (2011) Voltammetric determination of ethanol by using mushroom (Agaricus bisporus) tissue homogenate-based biosensor. Hacettepe Journal of Biology and Chemistry 39(1): 65-70.
Cate, D.M., Jaclyn, A.A., Mettakoonpitak, J., Henry, C.S. (2015) Recent Developments in Paper-Based Microfluidic Devices. Analytical Chemistry 87(1): 19-41.

Simpson, G. (1987) Accuracy and precision of breath-alcohol measurements for a random subject in the postabsorptive state. Clinical Chemistry 33(2 pt 1): 261-268.

Wu, Q., Cheng, S., Wang, C., Li, X., Li, Z., Hao, C. Magnetic porous carbon derived from a zinccobalt metal-organic framework: A adsorbent for magnetic solid phase extraction of flunitrazepam. Microchim Acta 1-9.

*Corresponding author: Tel: 0773311010; E-mail: mnkaumal@sci.cmb.ac.lk 